\title{
TYCHO ASTROMETRY AND PHOTOMETRY
}

\author{
E. HØG \\ University Observatory \\ 1350 Copenhagen K, Denmark
}

\begin{abstract}
In the Tycho project astrometric and photometric data of about 1000000 stars to a limit of $B=12 \mathrm{mag}$ will be derived. The brightest 500000 stars will obtain magnitudes in two colours $B_{T}$ and $V_{T}$, the fainter 500000 usually only one broad-band magnitude $T$. There is confidence that an accuracy will be achieved about 0.03 arcsec for positions and 0.03 mag for magnitudes, at $B=10.5 \mathrm{mag}$. The results are obtained by appropriate treatment of the continuous data records generated by the Hipparcos satellite's star mapper (which provide simultaneous measurements in two spectral channels), based on predicted star transits using its own 'Tycho Input Catalogue'. The data treatment is carried out by the Tycho Data Analysis Consortium (TDAC), using calibration and satellite attitude information from the two Hipparcos data reduction consortia. An overview of the data reductions is given. Raw observation data and their numerical treatment are described. This assessment is based on a small data set, i.e. 12 weeks spread over the first 8 months of the Tycho 'revised mission' where the satellite is in the elliptical transfer orbit, instead of the iniended geostationary orbit.
\end{abstract}

A paper on Tycho with the above abstract has been submitted for publication in Astronomy $\mathcal{E}$ Astrophysics on June 19, 1991 by Høg E., Bastian U., Egret D., Grewing M., Halbwachs J.L., Wicenec A., Bässgen G., Bernacca P.L., Donati F., Kovalevsky J., van Leeuwen F., Lindegren L., Pedersen H., Perryman M.A.C., Petersen C., Scales D., Snijders M.A.J., Wesselius P.R. 